\title{
Reduced continuous-flow left ventricular assist device speed does not decrease von Willebrand factor degradation
}

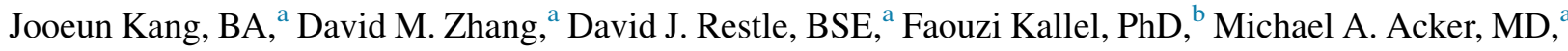 \\ Pavan Atluri, MD, ${ }^{a}$ and Carlo R. Bartoli, $\mathrm{MD}, \mathrm{PhD}^{\mathrm{a}}$
}

\begin{abstract}
Background: Nonsurgical bleeding is a frequent complication of continuousflow left ventricular assist device (LVAD) support. Abnormal von Willebrand factor (vWF) metabolism plays a major role. However, the relationship between LVAD speed and vWF degradation is unknown. Recent evidence has demonstrated that supraphysiologic shear stress from continuous-flow LVADs accelerates vWF degradation and causes an acquired vWF deficiency and bleeding. To manage LVAD-associated bleeding, it has been proposed that reduced LVAD speed may decrease shear stress and thereby reduce pathologic vWF metabolism. However, there are little published data to support this clinical practice. We tested the hypothesis that reduced continuous-flow LVAD speed decreases vWF degradation.
\end{abstract}

Methods: Whole blood was collected from patients before and after the implantation of a HeartMate II continuous-flow LVAD $(n=10)$ to quantify in vivo vWF degradation. In parallel, to evaluate the relationship between LVAD rpm and vWF degradation, whole blood was collected from human donors $(\mathrm{n}=30)$. Single-donor units of blood circulated in an ex vivo HeartMate II mock circulatory loop for 12 hours at 11,400,10,000, or $8600 \mathrm{rpm}(\mathrm{n}=10 /$ each rpm group). vWF multimers and degradation fragments were characterized with electrophoresis and immunoblot analysis. Paired Student $t$ tests were performed within each group. ANOVA with Tukey post hoc test was performed across groups.

Results: In patients, LVAD support reduced large vWF multimers and significantly $(P<.05)$ increased vWF degradation fragments. The profile of vWF degradation was nearly identical between LVAD patients and blood circulated in the LVAD mock circulatory loop. At 11,400, 10,000, and $8600 \mathrm{rpm}$, decreased large vWF multimers and significantly increased vWF degradation fragments were noted. vWF degradation fragments were not statistically different across the $3 \mathrm{rpm}$ groups or versus LVAD patients, which suggested that LVAD rpm did not influence vWF degradation.

Conclusions: Reduced LVAD speed (within the clinical operational range) did not significantly decrease vWF degradation in a mock circulatory loop with human blood. During bleeding events, reduced LVAD speed, itself, may not diminish vWF degradation. (J Thorac Cardiovasc Surg 2016;151:1747-54)

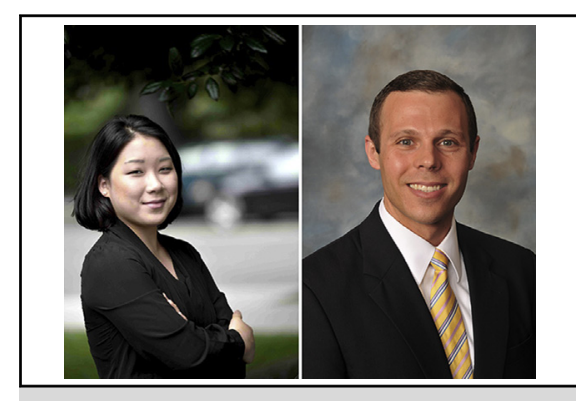

Jooeun Kang, BA, and Carlo R. Bartoli, MD, PhD

Central Message

Reduced LVAD speed does not decrease von Willebrand factor degradation and may be an inappropriate adjunctive intervention to manage LVAD-associated bleeding.

\section{Perspective}

Nonsurgical bleeding is a frequent complication during mechanical circulatory support. To manage LVAD-associated bleeding, it has been proposed that reduced LVAD speed may reduce the pathologic metabolism of von Willebrand factor. However, there are little published data to support this clinical practice. We report that reduced LVAD speed did not decrease von Willebrand factor degradation and might not be an appropriate management strategy for LVADassociated bleeding.

See Editorial Commentary page 1755.

\footnotetext{
From the a Division of Cardiovascular Surgery, Hospital of the University of Pennsylvania, Philadelphia, Pa; and ${ }^{\mathrm{b}}$ Thoratec Corporation, Pleasanton, Calif.

This study was performed with support from the University of Pennsylvania Clinical Translational Research Center. Thoratec Corporation provided HeartMate II LVADs for this study.

Received for publication Aug 26, 2015; revisions received Jan 9, 2016; accepted for publication Jan 15, 2016; available ahead of print March 11, 2016.

Address for reprints: Carlo R. Bartoli, MD, PhD, Hospital of the University of Pennsylvania, Division of Cardiovascular Surgery, Stemmler Hall, Room 351, Philadelphia, PA 19104 (E-mail: carlo.bartoli@uphs.upenn.edu). 0022-5223/\$36.00

Copyright (c) 2016 by The American Association for Thoracic Surgery http://dx.doi.org/10.1016/j.jtcvs.2016.01.031
}

Nonsurgical bleeding is a frequent complication of continuous-flow left ventricular assist device (LVAD) support. ${ }^{1}$ Thirty to $75 \%$ of LVAD patients bleed. ${ }^{2-6}$

Scanning this QR code will take you to supplemental table for this article.

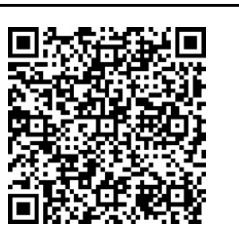




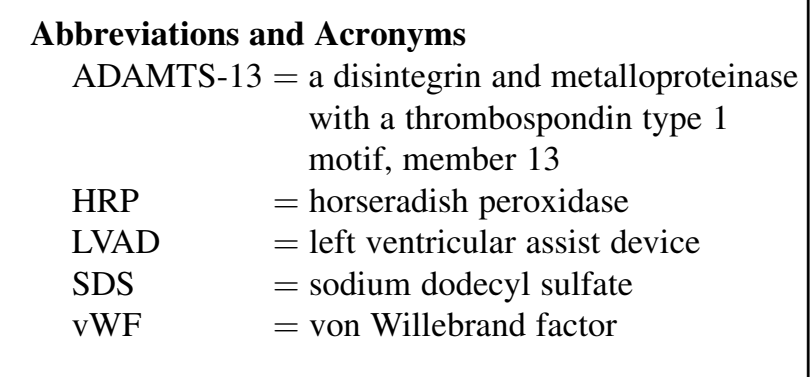

Abnormal von Willebrand factor (vWF) degradation plays a major role. Supraphysiologic shear stress from continuous-flow LVADs accelerates vWF metabolism and depletes functional vWF multimers. ${ }^{7}$ As a result, an acquired vWF deficiency predisposes LVAD patients to bleeding events. ${ }^{8}$

Recently, continuous-flow LVAD speed reduction has been reported as a management strategy for LVADassociated bleeding. It has been suggested that reduced LVAD speed may reduce vWF degradation and predisposition to bleeding. ${ }^{9-14}$ However, there are only limited clinical data and no experimental data to support this practice. Although reduced LVAD speed causes a modest decrease in shear stress, physiologic levels of shear stress cannot be achieved with LVAD speeds in the clinical operational range. ${ }^{15-17}$ Therefore, it is unlikely that a lower LVAD speed causes a biologically relevant reduction in shear stress or a reduction in pathologic vWF metabolism.
In order to verify this point, we tested the hypothesis that reduced LVAD speed reduces $\mathrm{VWF}$ degradation in a continuous-flow LVAD mock circulatory loop with human blood.

\section{METHODS}

This study was conducted with approval from the University of Pennsylvania's blood bank and Institutional Review Board (818944, "Novel Model of LVAD-Like von Willebrand Factor Degradation for the Investigation of Bleeding in Patients with an LVAD"). Subjects provided informed consent.

\section{Blood Sample Collection}

Paired blood samples were obtained from patients $(n=10$; mean age, $59 \pm 5$ years; range, 35 to 76 years) at baseline (before LVAD implantation) and at 7 days after implantation of a HeartMate II continuous-flow LVAD (Thoratec, Pleasanton, Calif). These samples were used to quantify in vivo vWF degradation in patients undergoing continuous-flow LVAD support.

In parallel, to evaluate the relationship between LVAD speed and vWF degradation, fresh whole blood was obtained from human donors $(\mathrm{n}=30)$ via venipuncture. Single-donor units of blood were circulated in an ex vivo HeartMate II mock circulatory loop for 12 hours at $11,400,10,000$, or $8600 \mathrm{rpm}$ ( $\mathrm{n}=10$ for each rpm group). Blood samples from LVAD patients were anticoagulated in buffered sodium heparin tubes (BD Biosciences, Franklin Lakes, NJ). Blood samples from volunteer donors were anticoagulated in citrate phosphate dextrose-adenine 1 blood collection bags (Blood Resources International, Dixon, Calif).

\section{Mock Circulatory Loop}

An ex vivo mock circulatory loop was constructed with approximately $75 \mathrm{~cm}$ of 3/8-inch connective Tygon tubing (Medtronic, Minneapolis,

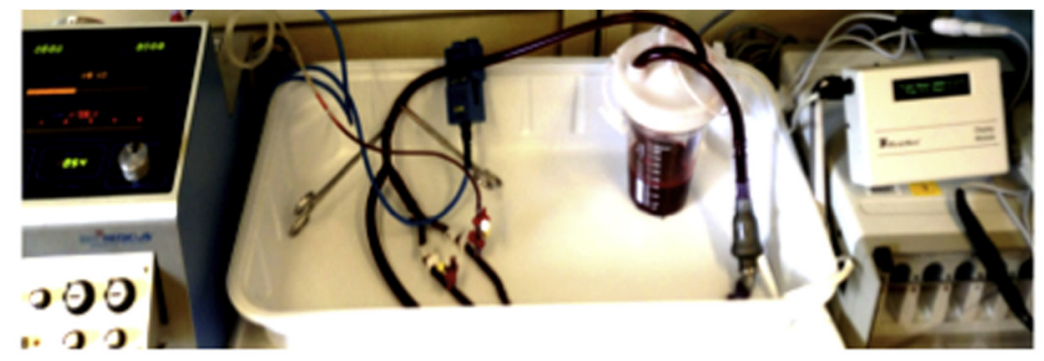

A 1. 20

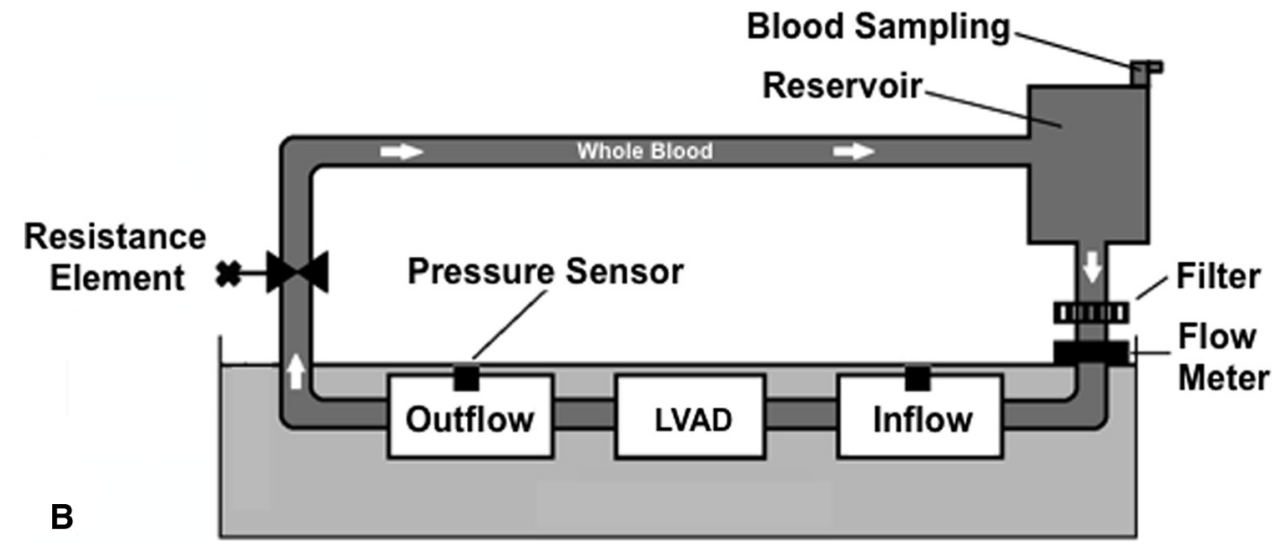

FIGURE 1. A, The LVAD mock circulatory loop. B, Schematic of the LVAD mock circulatory loop. LVAD, Left ventricular assist device. 


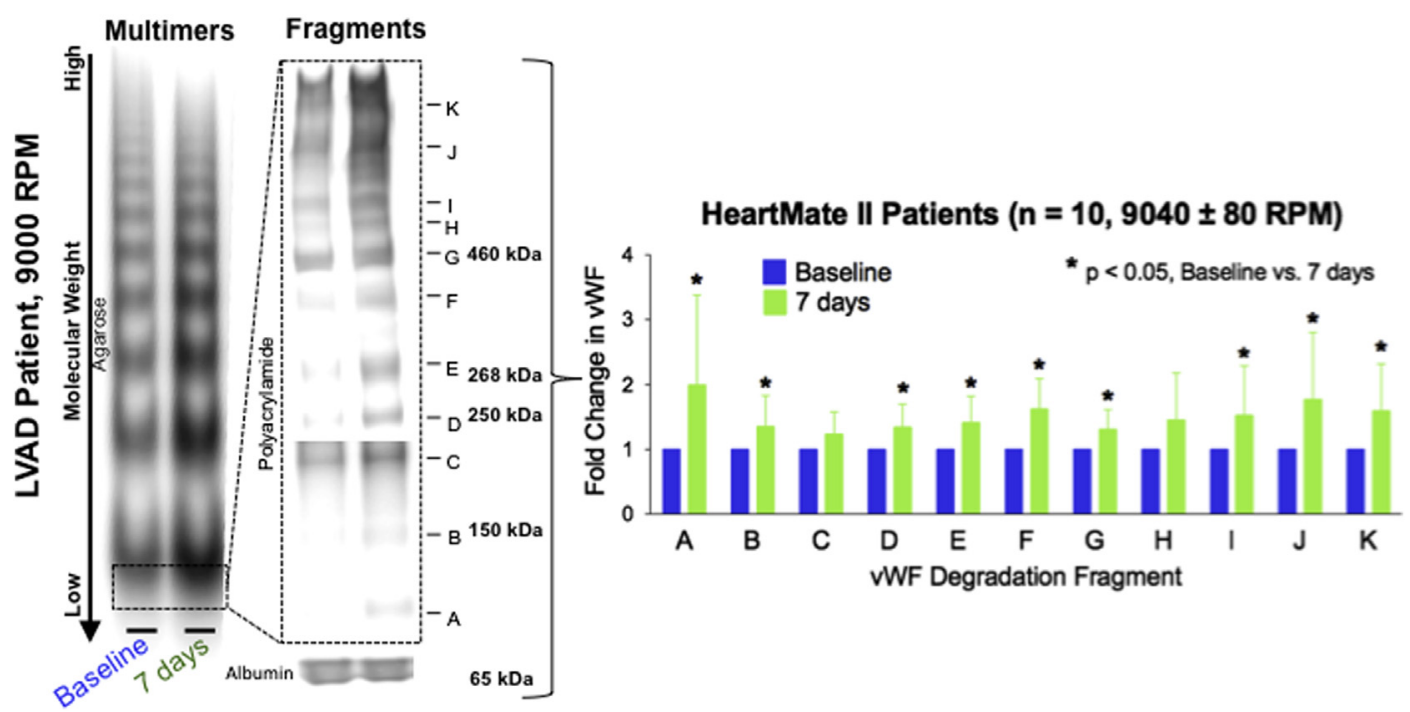

FIGURE 2. Paired blood samples from a single patient before and after HeartMate II continuous-flow LVAD implantation demonstrated reduced large vWF multimers and increased small vWF multimers (agarose blot), as well as significantly increased vWF degradation fragments (polyacrylamide blot). *Immmunoblots in Figures 2-5 represent duplicate samples studied with 2 different methods: (1) agarose gel electrophoresis to resolve high-molecularweight vWF multimers, and (2) polyacrylamide gel electrophoresis to resolve small vWF degradation fragments. LVAD, Left ventricular assist device; $v W F$, von Willebrand factor.

Minn) and a 1-L reservoir with a blood sampling port as previously described $^{18,19}$ (Figure 1). A HeartMate II continuous-flow LVAD was placed in series with this loop. The loop was primed with approximately 450 to $500 \mathrm{~mL}$ of whole human blood. A c-clamp resistor (Thermo Fisher Scientific, Pittsburgh, Pa) was placed distal to the LVAD to control pump head pressure and total loop flow. An ultrasonic flow probe (Transonics, Ithaca, NY) and fluid-filled pressure transducer (BD Biosciences) were placed to measure volumetric flow through the loop and pump outlet pressure, respectively. Loop resistance was modified as needed to maintain a physiologic pressure for each rpm group with a target mean pressure of 75 to $80 \mathrm{~mm} \mathrm{Hg}$. All experiments were performed at room temperature. Three sets of experiments with a different rpm $(11,400,10,000$, or $8600 \mathrm{rpm}, \mathrm{n}=10$ for each rpm group) were performed with separate units of blood.

\section{Agarose Gel Electrophoresis and Immunoblot}

\section{Analysis for High Molecular Weight vWF Multimers}

The relative size of high molecular weight plasma vWF multimers was analyzed by standard immunoblotting techniques as previously described. ${ }^{7,18-20}$ Plasma samples were each combined at a 1:20 ratio with loading buffer (1 mM EDTA, $10 \mathrm{mM}$ Tris- $\mathrm{HCl}, 8 \mathrm{M}$ urea, $2 \%$ sodium dodecyl sulfate [SDS], and bromophenol blue, $\mathrm{pH}$ 8.0). Samples were heated in a $60^{\circ} \mathrm{C}$ water bath for 20 minutes. Samples were loaded into $1.8 \%$ agarose-SDS gels $(8.3 \times 8 \mathrm{~cm} \times 1.5 \mathrm{~mm} ; 1 \%$ SDS, $0.375 \mathrm{M}$ Tris-HCl; Life Technologies, Grand Island, NY). Electrophoresis was performed at $60 \mathrm{~V}$ for 1.75 hours at $4^{\circ} \mathrm{C}$ in $1 \times$ Tris-Acetate SDS

TABLE 1. Mean blood flow and pressure during reduced revolutions per minute in a LVAD mock circulatory loop

\begin{tabular}{lcccc}
\hline & \multicolumn{3}{c}{ rpm } & \\
\cline { 2 - 4 } \multicolumn{1}{c}{ Variable } & $\mathbf{1 1 , 4 0 0}$ & $\mathbf{1 0 , 0 0 0}$ & $\mathbf{8 6 0 0}$ & $\boldsymbol{P}$ value \\
\hline Flow, $\mathrm{L} / \mathrm{min}$ & $>6.5$ & $4.3 \pm 0.2^{*}$ & $3.4 \pm 0.1^{*}, \dagger$ & $<.0001$ \\
Pressure, $\mathrm{mm} \mathrm{Hg}$ & $81 \pm 1$ & $76 \pm 3$ & $78 \pm 1$ & .20 \\
\hline
\end{tabular}

$* P<.001$ versus $11,400 \mathrm{rpm} . \dagger P<.001$ versus $10,000 \mathrm{rpm}$. running buffer (Life Technologies) in an XCell SureLock Mini-Cell Electrophoresis System (Life Technologies).

Proteins were transferred for 8.5 minutes with the iBlot dry transfer device onto iBlot PVDF Gel Transfer Stacks (Life Technologies). The resulting blots were blocked for 1 hour in milk blocking buffer $(5 \%$ dried milk powder in Tris-buffered saline [TBS] and $0.05 \%$ Tween-20; Thermo Fisher Scientific, Waltham, Mass). Blots were incubated with rabbit anti-human vWF primary antibody (1/500; Dako, Carpinteria, Calif) overnight at $4{ }^{\circ} \mathrm{C}$ in TBS-Tween $/ 5 \%$ dried milk powder. The next day, blots were washed in TBS-Tween and incubated with goat anti-rabbit IgG horseradish peroxidase (HRP)-conjugated secondary antibody (1/3000; Cell Signaling, Danvers, Mass) for 2 hours at room temperature in TBS-Tween $/ 5 \%$ dried milk powder. Finally, the blots were developed with Luminata Forte Western Blot HRP Substrate (Millipore, Billerica, Mass) and imaged with an ImageQuant LAS 4000 biomolecular imager (GE Healthcare Bio-Sciences, Pittsburgh, Pa).

\section{Polyacrylamide Gel Electrophoresis and Immunoblotting for vWF Degradation Fragments}

The relative size of plasma vWF degradation fragments was analyzed by standard immunoblotting techniques as previously described. ${ }^{7,18-20}$ Plasma samples were combined 1:40 in NuPAGE LDS Sample Buffer (Life Technologies). Samples were incubated at $70^{\circ} \mathrm{C}$ for 10 minutes and then loaded into NuPAGE $3 \%$ to $8 \%$ Tris-acetate gels on a prestained Precision Plus Protein Kaleidoscope Standard Protein Ladder (Bio-Rad, Hercules, Calif) and HiMark Prestained Standard Protein Ladder (Life Technologies). Electrophoresis was performed at $150 \mathrm{~V}$ for 1.25 hours at $4{ }^{\circ} \mathrm{C}$ with $1 \times$ Tris-acetate SDS running buffer (Life Technologies). Proteins were transferred, blocked, probed for vWF, and imaged as described above.

A protein loading control was performed by immunoblotting for human plasma albumin. Blots were washed and blocked for 1 hour at room temperature in TBS-Tween $/ 5 \%$ dried milk powder, incubated overnight at $4{ }^{\circ} \mathrm{C}$ in primary goat anti-human albumin HRP-conjugated antibody $(1 / 5000$ in TBS-Tween $/ 5 \%$ dried milk powder; Abcam, Cambridge, Mass), and then washed, developed, and imaged as described above. 


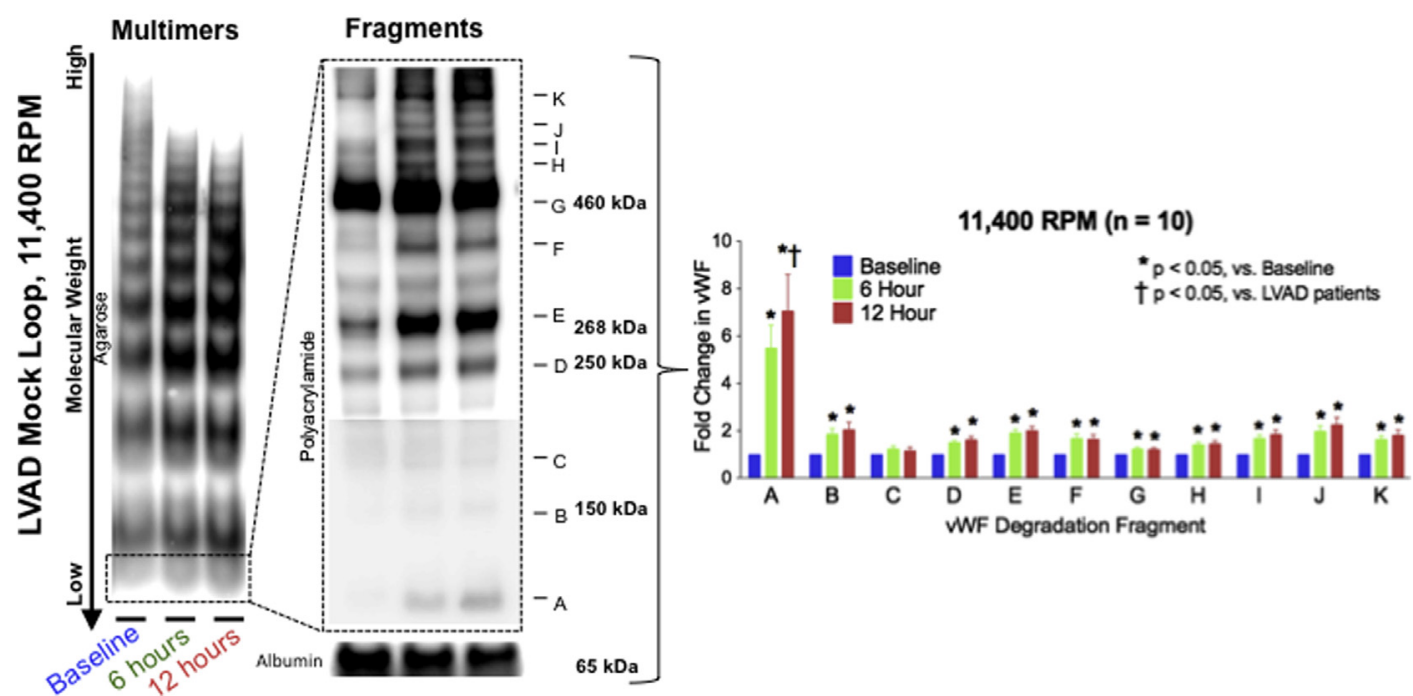

FIGURE 3. Human blood circulated through a HeartMate II mock circulatory loop at 11,400 rpm demonstrated significant vWF degradation. Compared with 10,000 and $8600 \mathrm{rpm}$, vWF degradation was not statistically different. $L V A D$, Left ventricular assist device; $v W F$, von Willebrand factor.

\section{vWF Degradation Fragment Immunoblot Analysis}

Relative levels of vWF fragments were quantified. Images containing paired samples from control and experimental groups underwent differential contrast enhancement to highlight biological changes, especially with the smallest vWF fragments (fragments A to C), which stain less prominently due to the relative size difference versus larger vWF fragments and small vWF multimers. Data were intentionally presented as fold increase to negate the possibility of varying contrast enhancement across multiple immunoblots. ImageJ (National Institutes of Health, Bethesda, $\mathrm{Md}$ ) was used to measure the chemiluminescent intensity of $11 \mathrm{vWF}$ fragment bands at 6 hours and at 12 hours.

\section{Statistics}

Prism version 5.00 (GraphPad Software, La Jolla, Calif) was used to perform statistical analyses and plot data. Paired Student $t$ tests compared levels of vWF degradation fragments between pre-LVAD and post-LVAD patient samples and between baseline and 6-hour or 12-hour samples from the LVAD mock circulatory loop. Nonrepeated measures ANOVA with Tukey post hoc test of means compared fold changes for each vWF degradation fragment from each group. A $P$ value $<.05$ (95\% confidence interval) was considered statistically significant. Presented $P$ values were raw and did not account for multiple comparisons. All data are presented as mean \pm standard error.

\section{RESULTS}

vWF Degradation and Bleeding in LVAD Patients

In patients, the average LVAD speed was $9040 \pm 80 \mathrm{rpm}$ (range, 8800 to $9600 \mathrm{rpm}$ ). LVAD support generated a common profile of $\mathrm{vWF}$ multimers and $\mathrm{vWF}$

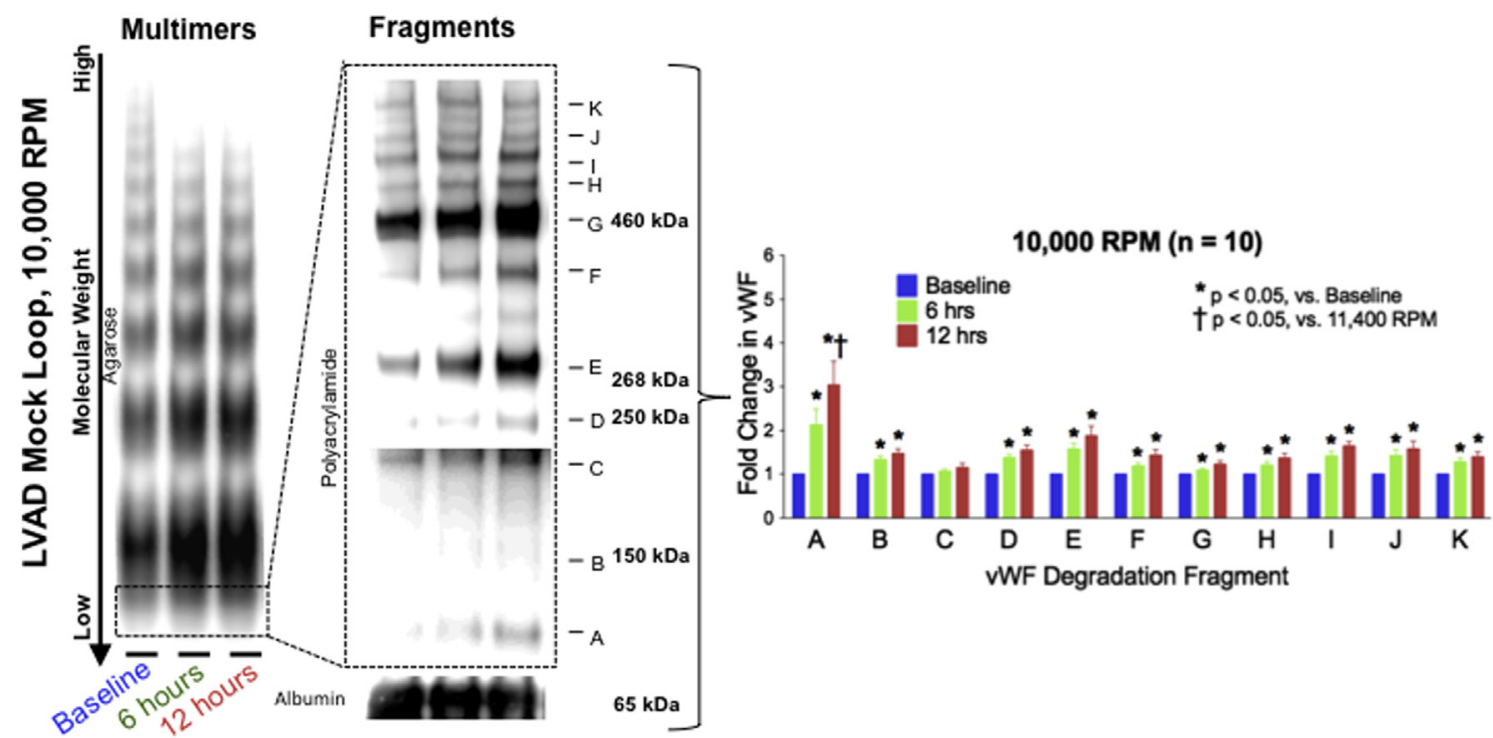

FIGURE 4. Human blood circulated through a HeartMate II mock circulatory loop at 10,000 rpm demonstrated significant vWF degradation. Compared with 11,400 and $8600 \mathrm{rpm}$, vWF degradation was not statistically different. $L V A D$, Left ventricular assist device; $v W F$, von Willebrand factor. 


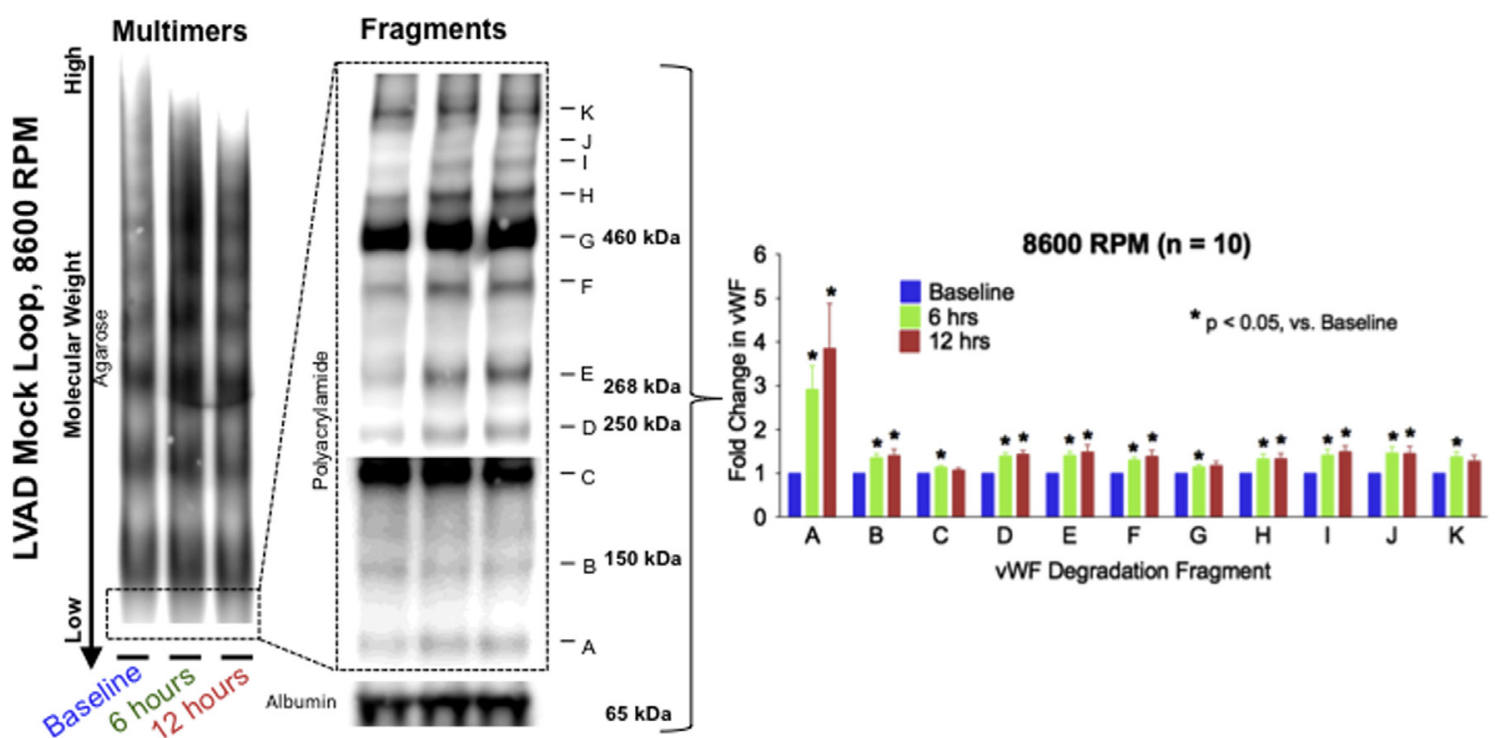

FIGURE 5. Human blood circulated through a HeartMate II mock circulatory loop at $8600 \mathrm{rpm}$ demonstrated significant vWF degradation. Compared with 11,400 and 10,000 rpm, vWF degradation was not statistically different. LVAD, Left ventricular assist device; $v W F$, von Willebrand factor.

degradation fragments. ${ }^{7,18}$ LVAD support reduced large vWF multimers, increased small vWF multimers, and significantly $(P<.05)$ increased 9 out of $11 \mathrm{vWF}$ degradation fragments (Figure 2).

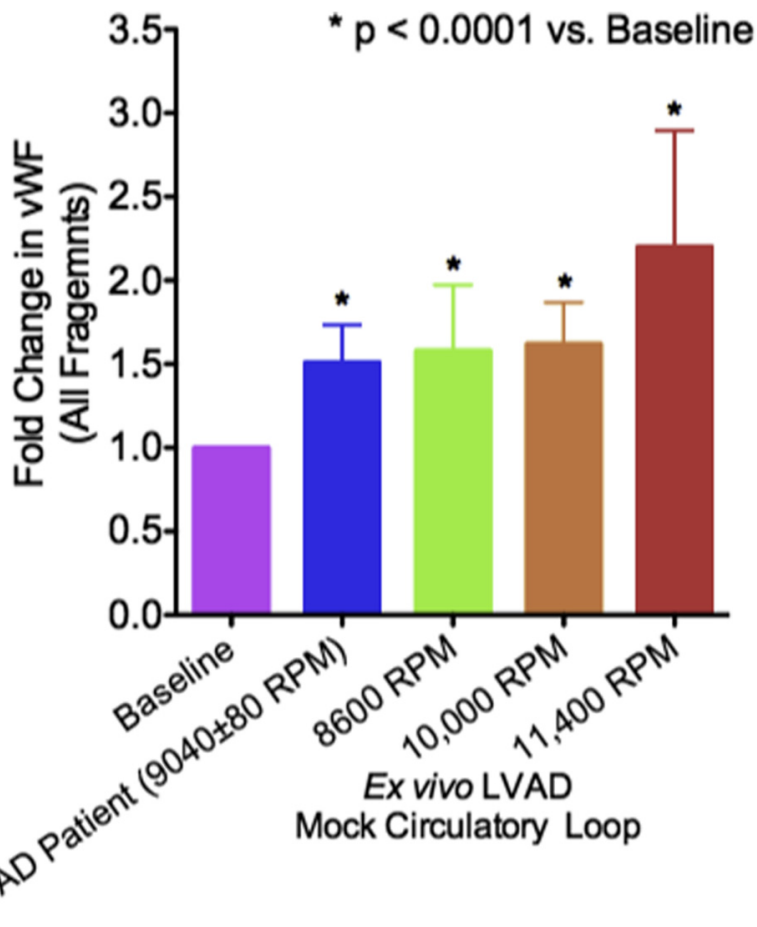

FIGURE 6. The total fold change for vWF degradation across all vWF degradation fragments in LVAD patients and in an LVAD mock circulatory loop with whole human blood during different rpm conditions. Fold change was significantly elevated between baseline and each group. Fold change was not significantly different across groups. $L V A D$, Left ventricular assist device; $v W F$, von Willebrand factor.
Clinically significant bleeding that resulted in hospitalization and an altered anticoagulation/antiplatelet regimen occurred in $60 \%$ of the 10 patients studied ( 4 gastrointestinal bleeds and 2 intracranial bleeds).

\section{vWF Degradation in the LVAD Mock Circulatory Loop}

Table 1 demonstrates the hemodynamic profile of each rpm group in the LVAD mock circulatory loop. As rpm decreased, flow decreased significantly $(P$ value across groups $<.0001$, ANOVA). Pressure was not statistically different across the 3 groups.

The profile of $\mathrm{vWF}$ degradation fragments was nearly identical between blood from LVAD patients and blood circulated through the LVAD mock circulatory loop. Reduced large vWF multimers, increased small vWF multimers, and significantly increased vWF degradation fragments were observed at 11,400, 10,000, and $8600 \mathrm{rpm}$ (Figures 3-5).

\section{vWF Degradation Was Similar Across Patients and Each rpm Group}

vWF degradation was not statistically different at 11,400 , 10,000 , and $8600 \mathrm{rpm}$ in the LVAD mock circulatory loop and in LVAD patients. ANOVA with Tukey post hoc test performed across rpm groups revealed that after 12 hours in the LVAD mock circulatory loop, vWF degradation for each vWF fragment was not statistically different across rpm groups with one exception. The A fragment was significantly greater in the $11,400 \mathrm{rpm}$ group compared with the LVAD patients at 7 days. 
Figure 6 and Table E1 present summary fold change data for each rpm group. Overall, fold change was significantly elevated between baseline and each group $(P<.0001)$ but was not significantly different across groups.

\section{DISCUSSION}

In this study, the speed of a continuous-flow LVAD (within the clinical operational range) did not influence vWF degradation. Specifically, we observed that (1) blood from continuous-flow LVAD patients and human blood circulated through a continuous-flow LVAD in a mock circulatory loop exhibited the same profile of $\mathrm{vWF}$ multimers and vWF degradation fragments, and (2) vWF degradation was not statistically different at 11,400 , 10,000 , and $8600 \mathrm{rpm}$. For the first time these data demonstrate that reduction of LVAD speed, itself, does not reduce vWF degradation.

\section{Supraphysiologic Shear Stress Causes Pathologic vWF Degradation and Predisposition to Bleeding}

vWF, a large multimeric glycoprotein, circulates in the plasma and plays a critical, early role in primary hemostasis. ${ }^{21}$ vWF metabolism is closely regulated by the rheological conditions of the blood. ${ }^{22}$ Shear stress is a powerful trigger for vWF metabolism by the vWF-specific protease, ADAMTS-13 (a disintegrin and metalloproteinase with a thrombospondin type 1 motif, member 13). ${ }^{22,23}$ During this process, large vWF multimers are degraded into small peptide fragments. ${ }^{24,25}$ As a result, the absence of large vWF multimers predisposes to bleeding.

Importantly, continuous-flow LVADs significantly elevate shear stress. ${ }^{12,18}$ With this in mind, we recently described the major mechanism of pathologic vWF degradation during LVAD support. ${ }^{7}$ Supraphysiologic shear stress from a continuous-flow LVAD accelerates vWF metabolism by ADAMTS-13. In this process, ADAMTS-13 cleaves large, biologically active vWF multimers into small vWF fragments with reduced hemostatic function. As a result, LVAD patients develop the same clinical behavior (ie, mucosal bleeding, gastrointestinal bleeding, prolonged bleeding time) and hematologic derangements (ie, reduced high-molecular-weight vWF multimers, reduced platelet aggregation through the ristocetin-vWF activation pathway, reduced $\mathrm{vWF}$ antigen, reduced $\mathrm{vWF}$ :collagen-binding activity) as a patient with congenital type II von Willebrand disease.

A similar clinical relationship between abnormal shear stress and degradation of vWF is well described. ${ }^{21}$ In patients with critical aortic stenosis, increased shear stress through the stenotic aortic valve reduces large vWF multimers. ${ }^{26}$ Within hours of aortic valve replacement (and reestablishment of normal shear stress), large vWF multimers return. These findings highlight in humans the clinical importance of physiologic processes that alter shear stress and thereby vWF metabolism.

\section{The Relationship Between LVAD Revolutions per Minute and vWF Degradation}

In this study, vWF degradation was similar in blood from LVAD patients and blood circulated through a continuous-flow LVAD at different rpms. In each group, decreased large vWF multimers and significantly increased vWF degradation fragments were noted. Interestingly, blood circulated through the mock loop at $11,400 \mathrm{rpm}$ produced significantly more vWF fragment A than was observed in LVAD patients. The A fragment represents the 140-kDa ADAMTS-13 cleavage fragment, which is the smallest vWF peptide. As such, A fragments are the direct product of ADAMTS-13 cleavage from the terminal ends of a vWF multimer. Biologically, this may suggest that generation of the A fragment is greater at high rpm (ie, highest shear stress) than at lower rpm (ie, relatively lower shear stress). The clinical relevance of this finding is unclear. A large vWF multimer $(\sim 20,000 \mathrm{kDa})$ that loses its terminal peptide tail $(140 \mathrm{kDa})$ is still a large, functional vWF multimer. Although interesting from a biological standpoint, it is unclear whether this finding has clinical significance.

\section{Clinical Effects of Reducing LVAD Speed}

In recent years, it had become a common practice to reduce the speed of continuous-flow LVADs to promote aortic valve opening and pulsatility. Potential benefits of pulsatility include favorable myocardial remodeling, prevention of aortic valve thrombosis, leaflet fusion, de novo aortic insufficiency, reduced arterial stiffening, better end-organ function, and, possibly, reduced bleeding. ${ }^{27}$ Indeed, recent literature contains statements with increasing frequency that LVAD speed reduction may be a management strategy for LVAD-associated bleeding. It has been suggested that lower LVAD speeds cause less vWF degradation and may reduce the predisposition to bleeding. ${ }^{9-14}$ However, there are only limited clinical data and no experimental data to support this practice. In fact, clinical observations have demonstrated that reduced continuous-flow LVAD speed does not influence vWF degradation ${ }^{13}$ and that pulsatility induced by decreasing LVAD speed may cause turbulent flow channels that promote thrombosis. ${ }^{28,29}$ As such, the trend in managing LVAD patients with lower pump speeds may have inadvertently contributed to the recent abrupt rise in LVAD thrombosis. ${ }^{30}$

Current continuous-flow LVADs generate shear stress 1 to 2 orders of magnitude greater than physiologic values. ${ }^{16}$ Of note, reduced LVAD speed causes a modest decrease in shear stress. However, this decrease in shear stress is biologically and clinically irrelevant. Within the clinical 


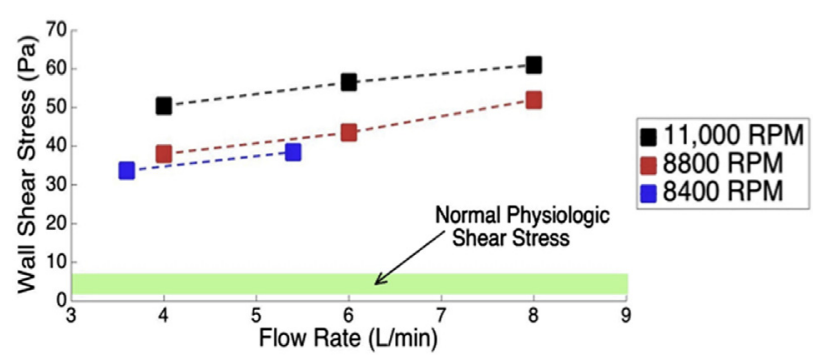

FIGURE 7. The relationship between HeartMate II speed and wall shear stress is shown at different rpms. The instantaneous shear stress at a single location between the impellar and pump housing was modeled by computational fluid modeling. Reduced LVAD speed produced a modest decrease in shear stress. However, speeds within the clinical operational range did not approach physiologic levels of shear stress. Thoratec Corporation independently generated and provided these data prior to this study.

operational range, the HeartMate II generates shear stress levels that far exceed physiologic values (Figure 7). For example, a decrease in rpm from 11,000 to 8800 reduces shear stress from 55 to $40 \mathrm{~Pa}$. However, this shear stress reduction does not reinstate a physiologic level of shear stress or even approach the physiologic range of normal shear stress, which is $<6 \mathrm{~Pa}$. Therefore, it is not surprising that reduced LVAD speed does not cause a biologically relevant reduction in pathologic vWF degradation.

There is, however, evidence that pulsatility protects against LVAD-associated bleeding. ${ }^{12}$ The mechanism(s) by which pulsatility reduces bleeding are unclear. A reduction in LVAD speed reduces left ventricular unloading. ${ }^{31}$ As a result, a reloaded left ventricle contains sufficient preload to open the aortic valve and generate an arterial pulse wave. We speculate that it is not pulsatility itself that reduces bleeding, but rather that opening of the aortic valve introduces a second blood flow path with a normal shear stress profile in parallel with flow through the LVAD. As a result, the volume of blood that is ejected through the aortic valve does not encounter supraphysiologic shear stress from the LVAD but rather encounters a normal physiologic flow profile, which does not degrade vWF during that circulatory cycle. Less total vWF degradation (and blood trauma) occurs, and patients are less predisposed to episodes of bleeding. This hypothesis is conceptually appealing but was not specifically tested in this investigation.

Of note, there are data that indicate that pulsatility during LVAD support does not reduce bleeding. Patients undergoing support with the CircuLite Synergy Pocket Micromed Pump, a partial-support continuous-flow LVAD that preserves native pulsatility, demonstrated a high rate of bleeding despite a normal pulse pressure. ${ }^{32}$ These data suggest that pulsatility, itself, does not protect against bleeding, and that a second flow path through the aortic valve may also not reduce LVAD-associated bleeding. Therefore, it still remains to be determined whether pulsatility or induced pulsatility with reduced LVAD speed favorably affects predisposition to bleeding, and/or whether this circulatory support strategy promotes thrombosis.

\section{Limitations}

Our experiments were performed in an ex vivo mock circulatory loop that does not recreate native anatomy in which blood flows through the LVAD in parallel with the left ventricle-to-aorta pathway. As such, we did not address the relationship between reduced LVAD speed and the proportion of blood flow through the native pathway that circumvents exposure to supraphysiologic shear stress. An unanswered question remains whether flow through this pathway is a mechanism to reduce vWF degradation (and bleeding).

The selected time points for blood analysis differed between the LVAD patients and the ex vivo mock circulatory loop experiments. Within 24 hours of initiation of LVAD support, large vWF multimers decrease. ${ }^{33}$ After 1 week of LVAD support, the effect plateaus and stabilizes. In our laboratory, we have observed clear evidence of early vWF degradation as early as 2 to 3 hours after the initiation of LVAD support. ${ }^{19}$ These findings suggest that within hours of exposure to supraphysiologic shear stress, vWF degradation begins. Over the course of hours to days, this effect reaches a steady-state degradation, which is counterbalanced by replenishment of vWF by endothelial cells. Importantly, our avascular LVAD mock circulatory loop does not contain endothelial cells. Therefore, it was not possible to evaluate the time course to steady-state $\mathrm{vWF}$ degradation with vWF replenishment that occurs in vivo in LVAD patients.

The effect of the artificial surface of the loop on vWF degradation may be considered a limitation of this study. In early experiments, we specifically tested the hypothesis that blood contact with the artificial interior of the loop affected vWF metabolism by including a non-LVAD mock loop control group in which blood was gently rocked for 12 hours in a laboratory agitator (unpublished data). Immunoblotting and platelet aggregation studies demonstrated no statistically significant differences in vWF degradation between baseline samples and the 12-hour non-LVAD control samples. This finding suggested that the artificial interior surface of the loop did not influence vWF degradation of our results. Nonetheless, blood contact with an artificial surface activates numerous pathways that alter the coagulation cascade, platelet function, and blood-protein interactions that may have important implications for LVAD therapy and may be a limitation of our model. 


\section{CONCLUSIONS}

Reduced LVAD speed (within the clinical operational range) did not reduce vWF degradation in a continuousflow LVAD mock circulatory loop with human blood. For the first time, these data suggest that reduced LVAD speed, itself, does not reduce vWF degradation.

\section{Conflict of Interest Statement}

F.K. was the Director of Clinical Studies at Thoratec Corporation during this investigation. P.A. reports consultant work for HeartWare. All other authors have nothing to disclose with regard to commercial support.

We thank Robert Dowling, MD, for providing valuable feedback during the study and drafting of the manuscript. We also thank Thoratec Corporation for donating the HeartMate II LVADs for the study. Ms Jessica Howard was instrumental in obtaining institutional review board approval for patient consent.

\section{References}

1. Kirklin JK, Naftel DC, Pagani FD, Kormos RL, Stevenson LW, Blume ED, et al. Sixth INTERMACS annual report: a 10,000-patient database. J Heart Lung Transplant. 2014;33:555-64.

2. Meyer AL, Malehsa D, Bara C, Budde U, Slaughter MS, Haverich A, et al. Acquired von Willebrand syndrome in patients with an axial flow left ventricular assist device. Circ Heart Fail. 2010;3:675-81.

3. Crow S, Milano C, Joyce L, Chen D, Arepally G, Bowles D, et al. Comparative analysis of von Willebrand factor profiles in pulsatile and continuous left ventricular assist device recipients. ASAIO J. 2010;56:441-5.

4. Uriel N, Pak SW, Jorde UP, Jude B, Susen S, Vincentelli A, et al. Acquired von Willebrand syndrome after continuous-flow mechanical device support contributes to a high prevalence of bleeding during long-term support and at the time of transplantation. J Am Coll Cardiol. 2010;56:1207-13.

5. Klovaite J, Gustafsson F, Mortensen SA, Sander K, Nielsen LB. Severely impaired von Willebrand factor-dependent platelet aggregation in patients with a continuous-flow left ventricular assist device (HeartMate II). J Am Coll Cardiol. 2009;53:2162-7.

6. Crow S, Chen D, Milano C, Thomas W, Joyce L, Piacentino V III, et al. Acquired von Willebrand syndrome in continuous-flow ventricular assist device recipients. Ann Thorac Surg. 2010;90:1263-9.

7. Bartoli CR, Restle DJ, Zhang DM, Acker MA, Atluri P. Pathologic von Willebrand factor degradation with a left ventricular assist device occurs via two distinct mechanisms: mechanical demolition and enzymatic cleavage. J Thorac Cardiovasc Surg. 2015;149:281-9.

8. Islam S, Cevik C, Madonna R, Frandah W, Islam E, Islam S, et al. Left ventricular assist devices and gastrointestinal bleeding: a narrative review of case reports and case series. Clin Cardiol. 2013;36:190-200.

9. Hayes HM, Dembo LG, Larbalestier R, O’Driscoll G. Management options to treat gastrointestinal bleeding in patients supported on rotary left ventricular assist devices: a single-center experience. Artif Organs. 2010;34:703-6.

10. Aggarwal A, Pant R, Kumar S, Sharma P, Gallagher C, Tatooles AJ, et al. Incidence and management of gastrointestinal bleeding with continuous flow assist devices. Ann Thorac Surg. 2012;93:1534-40.

11. Letsou GV, Shah N, Gregoric ID, Myers TJ, Delgado R, Frazier OH. Gastrointestinal bleeding from arteriovenous malformations in patients supported by the Jarvik 2000 axial-flow left ventricular assist device. J Heart Lung Transplant. 2005;24:105-9.

12. Wever-Pinzon O, Selzman CH, Drakos SG, Saidi A, Stoddard GJ, Gilbert EM, et al. Pulsatility and the risk of nonsurgical bleeding in patients supported with the continuous-flow left ventricular assist device HeartMate II. Circ Heart Fail. 2013;6:517-26.

13. Meyer AL, Malehsa D, Budde U, Bara C, Haverich A, Strueber M. Acquired von Willebrand syndrome in patients with a centrifugal or axial continuous flow left ventricular assist device. JACC Heart Fail. 2014;2:141-5.
14. Crow SS, Joyce DD. Are centrifugal ventricular assist devices the answer to reducing post-implantation gastrointestinal bleeding? JACC Heart Fail. 2014; 2:146-7.

15. Selgrade BP, Truskey GA. Computational fluid dynamics analysis to determine shear stresses and rates in a centrifugal left ventricular assist device. Artif Organs. 2012;36:E89-96.

16. Fraser KH, Zhang T, Taskin ME, Griffith BP, Wu ZJ. A quantitative comparison of mechanical blood damage parameters in rotary ventricular assist devices: shear stress, exposure time and hemolysis index. J Biomech Eng. 2012;134: 081002.

17. Kang J, Zhang DM, Restle DJ, Kallel F, Acker MA, Atluri P, et al. Reduced continuous-flow LVAD speed does not decrease von Willebrand factor degradation [abstract]. J Heart Lung Transplant. 2015;34:S209.

18. Restle DJ, Zhang DM, Hung G, Howard JL, Kallel F, Acker MA, et al. Preclinical models for translational investigations of left ventricular assist device-associated von Willebrand factor degradation. Artif Organs. 2015;39: 569-75.

19. Dassanayaka S, Slaughter MS, Bartoli CR. Mechanistic pathway(s) of acquired von Willebrand syndrome with a continuous-flow ventricular assist device: in vitro findings. ASAIO J. 2013;59:123-9.

20. Bartoli CR, Dassanayaka S, Brittian KR, Luckett A, Sithu S, Siess T, et al. Insights into the mechanism(s) of von Willebrand factor degradation during mechanical circulatory support. J Thorac Cardiovasc Surg. 2014;147: 1634-43.

21. Sadler JE. Biochemistry and genetics of von Willebrand factor. Annu Rev Biochem. 1998;67:395-424.

22. Zhang X, Halvorsen K, Zhang CZ, Wong WP, Springer TA. Mechanoenzymatic cleavage of the ultralarge vascular protein von Willebrand factor. Science. 2009; 324:1330-4.

23. Tsai HM, Sussman II, Nagel RL. Shear stress enhances the proteolysis of von Willebrand factor in normal plasma. Blood. 1994;83:2171-9.

24. Dent JA, Galbusera M, Ruggeri ZM. Heterogeneity of plasma von Willebrand factor multimers resulting from proteolysis of the constituent subunit. J Clin Invest. 1991;88:774-82.

25. Turner NA, Nolasco L, Ruggeri ZM, Moake JL. Endothelial cell ADAMTS-13 and VWF: production, release, and VWF string cleavage. Blood. 2009;114: 5102-11.

26. Vincentelli A, Susen S, Le Tourneau T, Six I, Fabre O, Juthier F, et al. Acquired von Willebrand syndrome in aortic stenosis. N Engl J Med. 2003; 349:343-9.

27. Bartoli CR, Atluri P. Do patients with a continuous-flow left ventricular assist device benefit from induced-pulsatility, or are we just spinning our wheels? J Thorac Cardiovasc Surg. 2015;150:945-6.

28. Wong K, Samaroo G, Ling I, Dembitsky W, Adamson R, del Alamo JC, et al. Intraventricular flow patterns and stasis in the LVAD-assisted heart. $J$ Biomech. 2014;47:1485-94.

29. Yang F, Kormos RL, Antaki JF. High-speed visualization of disturbed pathlines in axial flow ventricular assist device under pulsatile conditions. J Thorac Cardiovasc Surg. 2015;150:938-44.

30. Starling RC, Moazami N, Silvestry SC, Ewald G, Rogers JG, Milano CA, et al. Unexpected abrupt increase in left ventricular assist device thrombosis. $N$ Engl J Med. 2014;370:33-40.

31. Bartoli CR, Giridharan GA, Litwak KN, Sobieski M, Prabhu SD, Slaughter MS, et al. Hemodynamic responses to continuous versus pulsatile mechanical unloading of the failing left ventricle. ASAIO J. 2010;56:410-6.

32. Goda M, Jacobs S, Rega F, Peerlinck K, Jacquemin M, Droogne W, et al. Time course of acquired von Willebrand disease associated with two types of continuous-flow left ventricular assist devices: HeartMate II and CircuLite Synergy Pocket Micro-Pump. J Heart Lung Transplant. 2013; 32:539-45.

33. Heilmann C, Geisen U, Beyersdorf F, Nakamura L, Trummer G, BerchtoldHerz M, et al. Acquired von Willebrand syndrome is an early-onset problem in ventricular assist device patients. Eur J Cardiothorac Surg. 2011;40: 1328-33.

Key Words: bleeding, heart failure, left ventricular assist device, mechanical circulatory support, shear stress, von Willebrand factor 
TABLE E1. Von Willebrand factor degradation fold-change during varying revolutions per minute with the HeartMate II LVAD

\begin{tabular}{|c|c|c|c|c|c|c|c|c|c|c|c|c|c|}
\hline \multirow[b]{2}{*}{ rpm } & \multirow[b]{2}{*}{ Comparison } & \multicolumn{12}{|c|}{ von Willebrand factor degradation fragment } \\
\hline & & n & $\mathbf{A}$ & B & $\mathbf{C}$ & D & $\mathbf{E}$ & $\mathbf{F}$ & G & $\mathbf{H}$ & $\mathbf{I}$ & $\mathbf{J}$ & $\mathbf{K}$ \\
\hline \multicolumn{14}{|l|}{ LVAD patients } \\
\hline $9040 \pm 80$ & Pre-LVAD vs post-LVAD & 10 & $1.99 *$ & $1.35^{*}$ & 1.23 & $1.34 *$ & $1.41^{*}$ & $1.62 *$ & $1.31 *$ & 1.45 & $1.53^{*}$ & $1.77 *$ & 1.59 \\
\hline \multicolumn{14}{|c|}{ LVAD mock circulatory loop } \\
\hline \multirow[t]{2}{*}{8600} & Baseline vs 6 hour & 10 & $2.91 *$ & $1.36^{*}$ & $1.14^{*}$ & $1.39 *$ & $1.40^{*}$ & $1.30^{*}$ & $1.14 *$ & $1.33^{*}$ & $1.41^{*}$ & $1.46^{*}$ & $1.37 *$ \\
\hline & Baseline vs 12 hour & 10 & $3.85^{*}$ & $1.40 *$ & 1.08 & $1.43^{*}$ & $1.49^{*}$ & $1.39 *$ & 1.18 & $1.33^{*}$ & $1.49^{*}$ & $1.45^{*}$ & 1.28 \\
\hline \multirow[t]{2}{*}{10,000} & Baseline vs 6 hour & 10 & $2.13 *$ & $1.34 *$ & 1.07 & $1.38 *$ & $1.57^{*}$ & $1.19^{*}$ & $1.09 *$ & $1.21 *$ & $1.41^{*}$ & $1.43^{*}$ & 1.28 \\
\hline & Baseline vs 12 hour & 10 & $3.05^{*}$ & $1.48^{*}$ & 1.15 & $1.55^{*}$ & $1.88^{*}$ & $1.44 *$ & $1.23 *$ & $1.37 *$ & $1.65^{*}$ & $1.59^{*}$ & $1.40^{*}$ \\
\hline \multirow[t]{2}{*}{11,400} & Baseline vs 6 hour & 10 & $5.49 *$ & $1.86^{*}$ & 1.25 & $1.52 *$ & $1.91^{*}$ & $1.68^{*}$ & $1.24 *$ & $1.42 *$ & $1.68 *$ & $1.98 *$ & $1.64 *$ \\
\hline & Baseline vs 12 hour & 10 & $7.06^{*}$ & $2.04 *$ & 1.16 & $1.63 *$ & $2.01 *$ & $1.66^{*}$ & $1.22 *$ & $1.45^{*}$ & $1.85^{*}$ & $2.26^{*}$ & $1.82 *$ \\
\hline
\end{tabular}

LVAD, Left ventricular assist device. $* P<.05$ versus baseline. 